\title{
Patient Base
}

National Cancer Institute

\section{Source}

National Cancer Institute. Patient Base. NCI Thesaurus. Code C52644.

The total number of patients that have been evaluated or treated. 